\title{
Neutrino Masses and Solar Neutrinos
}

\author{
L. Wolfenstein \\ Carnegic Mellon University \\ Department of Physics \\ Pittsburgh, PA 15213
}

\begin{abstract}
It has been pointed out by Bahcall and Bethe and others that all solar neutrino data can be explained by MSW oscillations with $m\left(\nu_{1}\right) \sim 10^{-3}$ ev consistent with ideas of grand unified theories (GUTS). We point out here a second possibility consistent with GUTS ideas with $m\left(\nu_{\tau}\right) \sim 10^{-2} e v$ and $m\left(\nu_{\mu}\right) \sim 10^{-4} e v$. The two cases can be distinguished by a measurement of the solar neutrinos from ' $B e$.

\section{DISCLAIMER}

This report was prepared as an account of work sponsored by an agency of the United States Government. Neither the United States Government nor any agency thereof, nor any of their employees, makes any warranty, express or implied, or assumes any legal liability or responsibility for the accuracy, completeness, or usefulness of any information, apparatus, product, or process disclosed, or represents that its use would not infringe privately owned rights. Reference herein to any specific commercial product, process, or service by trade name, trademark, manufacturer, or otherwise does not necessarily constitute or imply its endorsement, recommendation, or favoring by the United States Government or any agency thereof. The views and opinions of authors expressed herein do not necessarily state or reflect those of the United States Government or any agency thereof.
\end{abstract}


There exists some indication that all the solar neutrinos of the $\nu_{r}$ variety produced in the deep interior of the sun do not reach delectors on earth. A particularly attractive solution to this apparent problem is that some of the $\nu_{t}$ are transformer to $\nu_{\mu}$ or $\nu_{\tau}$ on their way from the center to the surface of the sun by neutrino oscillations in matter, the MSW effect.

Several recent papers [1-4] have pointed to a particular choice of neutrino masses and mixings that fits all the experimental data perfectly and also fits into the theoretical ideas of grand unified theories (GUTS) like $\mathrm{SO}(10)$. Here we wish to point out an alternative choice that we feel is equally attractive.

The starting point of the recent papers is the theoretical neutrino flux of Bahcall and collaborators [5]. The Kamiokande detector finds [6] a flux abont, one-half of the theory and the Davis " $\mathrm{Cl}$ detector [7] a flux about 0.3 of the theory. Furthermore the first data [8] on a gallium experiment, sensitive to the low-energy pp neutrinos finds a rate less than half of the theoretical rate. All this data is fitted perfectly [1-4] by the MSW effect with a small mixing angle

$$
\begin{aligned}
& m\left(\nu_{x}\right) \approx 2 \times 10^{-3} \text { ev to } 4 \times 10^{-4} \text { ev } \\
& \sin ^{2} 2 \theta_{e x}=10^{-2} \text { to } 3 \times 10^{-1}
\end{aligned}
$$

where $\nu_{x}$ is $\nu_{\mu}$ or $\nu_{r}, \theta_{c x}$ is the mixing of $\nu_{r}$ with $\nu_{x}$, and one assumes $m\left(\nu_{r}\right) \ll m\left(\nu_{x}\right)$.

The suggestion from GUTS is the see-saw formula

$$
m\left(\nu_{x}\right) \sim 0.1 \frac{m^{2}\left(u_{: r}\right)}{M_{x}}
$$

where $m\left(u_{x}\right)$ is the mass of the $t$ quark (c quark) for $\nu_{x}=\nu_{\tau}\left(\nu_{\mu}\right), M_{x}$ is a large mass scale less t! 1 an or equal to the GUT scale. In particular, it may be an intermediate mass scale needed in theories like $\mathrm{SO}(10)$ in order that the theory fits the observed low-energy couplings. For the fit of Eq. (1), $M_{x} \sim 10^{15}$ Gev for $\nu_{x}=\nu_{\tau}$ and $M_{x} \sim 10^{\prime 1}$ Gev for $\nu_{x}=\nu_{\mu}$. Furthermore GUTS suggest that qualitatively the mixing angles should be like that of the quarks; thus

$$
\begin{aligned}
& \sin ^{2} 2 \theta_{\text {en }} \sim 10^{-1} \\
& \sin ^{2} 2 \theta_{c \tau} \sim 10^{-3} \text { to } 10^{-4}
\end{aligned}
$$

Thus there is some preference for choosing $\nu_{x}=\nu_{\mu}$ in this scenario, as suggested in Ref. 1.

It follows from Eq. (2) that neutrinos follow a mass hierarchy like the quarks. 
The relation between the $M_{x:}$ of different generations depends on the details of the theory. We consider two possibilities

$$
\begin{array}{ll}
\text { A. } \quad M_{x:}=\text { constant } \\
& m\left(\nu_{x}\right) \alpha m^{2}\left(u_{x}\right) \\
\text { B. } \quad M_{x}=\text { constant } \mathrm{xm}\left(u_{s}\right) \\
m\left(\nu_{x}\right) \alpha m\left(u_{x}\right)
\end{array}
$$

We do not believe it is possible to choose between these until one has a theory of the generation hierarchy.

To set the stage for our scenario we assume the data compared to the standard solar model (SSM) is given by

$$
\begin{array}{ll}
\text { Kamiokande/SSM } & =0.5 \pm 0.1 \\
{ }^{3} \mathrm{Cl} / S S M & =0.3 \pm 0.1 \\
\text { Gallium } / S S M & \leq 0.5
\end{array}
$$

We have enlarged the error on the ${ }^{37} \mathrm{Cl}$ experiment to allow for systematic errors which may be indicated by the fluctuation in the data. (As in Ref. 1-4 we attach no significance to the time variation in the neutrino flux reaching the detectors.)

Ignoring the gallium and ${ }^{37} \mathrm{Cl}$ data for the moment we fit the Kamiokande data with

$$
\begin{aligned}
& m\left(\nu_{\tau}\right) \approx 10^{-2} e v \\
& \sin ^{2} 2 \theta_{e \tau} \approx 3 \times 10^{-4}
\end{aligned}
$$

consistent with the analysis of Ref. 9. This is not a good fit to the Davis data because for this value of $\Delta m^{2}$ there is no suppression of the lower energy neutrinos (such as those from ${ }^{\top} \mathrm{Be}$ ) which provide one-quarter of the theoretically-expected ${ }^{37} \mathrm{Cl}$ signal. Thus this solution by itself predicts less suppression for ${ }^{37} \mathrm{Cl}$ than for Kamiokande. Furthermore it provides no suppression of the gallium signal.

However we now add $\nu_{\mu}$ to the story. We expect $m\left(\nu_{\mu}\right)$ to be much smaller than $m\left(\nu_{\tau}\right)$ with a much larger mixing angle. Assuming $\theta_{c \mu}$ is given by the Cabibbo angle and using Eq. (3b) which assumes $M_{x}$ scales with the generation we have

$$
\begin{aligned}
& m\left(\nu_{\mu}\right) \approx 10^{-1} e v \times(0.7-1.4) \\
& \sin ^{2} 2 \theta_{\text {e. }}=0.2
\end{aligned}
$$


This leads to a prediction of 30-70 SNU for the gallium experiment. In this scenario it is the $\nu_{r}-\nu_{\tau}$ transformation that converts ${ }^{8} B$ neutrinos and $\nu_{c}-\nu_{1}$, that converts the low energy pp and ${ }^{\top} B e$ neutrinos[10]. Because of this conversion of the ${ }^{7} B e$ neutrinos there is a further suppression of the ${ }^{3 i} \mathrm{Cl}$ signal thus making the Davis and Kamiokande results compatible within our assumed errors, particularly for the upper part of the range of $m\left(\nu_{n}\right)$ in $\mathrm{Eq}$. (6).

There exists an interesting way to distinguish these two choices of MSW solutions. In the case of Eq. (1) the gallium suppression corresponds to the adiabatic solution so that the suppression is increased as the energy increases. Thus for this solution a suppression of the gallium result by a factor of four involves an almost total suppression of the ${ }^{\top} B e$ neutrinos. In this case the gallium signal is nearly all due to pp neutrinos. In contrast for the case of Eqs. (5) and (6) the gallium suppression corresponds to the non-adiabatic solution for which the suppression decreases slowly as the energy increases. Thus the ${ }^{\top} B e$ neutrino suppression is always less than that of gallium. Indeed for this case the ${ }^{\top} B e$ neutrinos may contribute more than the pp neutrinos to the gallium signal. An experiment that directly detects the ${ }^{7} B e$ neutrinos, like the Borexino proposal, could thus distinguish these two solutions.

It should be emphasized that our use of GUTS is for qualitative guidance; specific models allow a large range of mass ratios and mixing angles [11]. We also believe that better quantitative observational data is needed before any definitive conclusions are possible.

This work was supported in part by the Department of Energy Contract No. DEAC02-76ER03066. I want to thank the D.O.E. Institute for Nuclear Theory of the University of Washington, where this work was completed, for its hospitality.

\section{References}

1. H.A. Bethe and J.N. Bahcall, Phys. Rev. D44, 2962 (1991); Phys. Rev. Lett. $65,2233(1990)$.

2. A.J. Baltz and J. Weneser, Phys. Rev. Lett. 66, 520 (1991).

3. S. Bludman et al, University of Pennsylvania preprint UPR09437, April 1991.

4. V. Barger et al, Phys. Rev. D43, 1110 (1991).

5. J.N. Bahcall and R.K. Ulrich, Rev. Mod. Phys. 60, 297 (1988). 
6. K.S. Ilirata et al, Phys. Rev. Lett. 65, 1297 (1990.

7. R. Davis, A,.K. Mann, and L. Wolfenstein, Ann. Rev. Nuc. Sci. 39, 467 (1989).

8. A.l. Abazov et al, Phys. Rev. Lett. 67, 3332 (i991).

9. K.S. Hirala et al, Phys. Rev. Letl. 65, 1301 (1990).

10. This possibility is discussed already in Neutrino 86 (eds. T. Kitagaki and $H$. Yuta), p. 12, World Scientific (1986).

11. For some examples see M. Gronau et al, Phys. Rev. D37, 2597 (1988). 

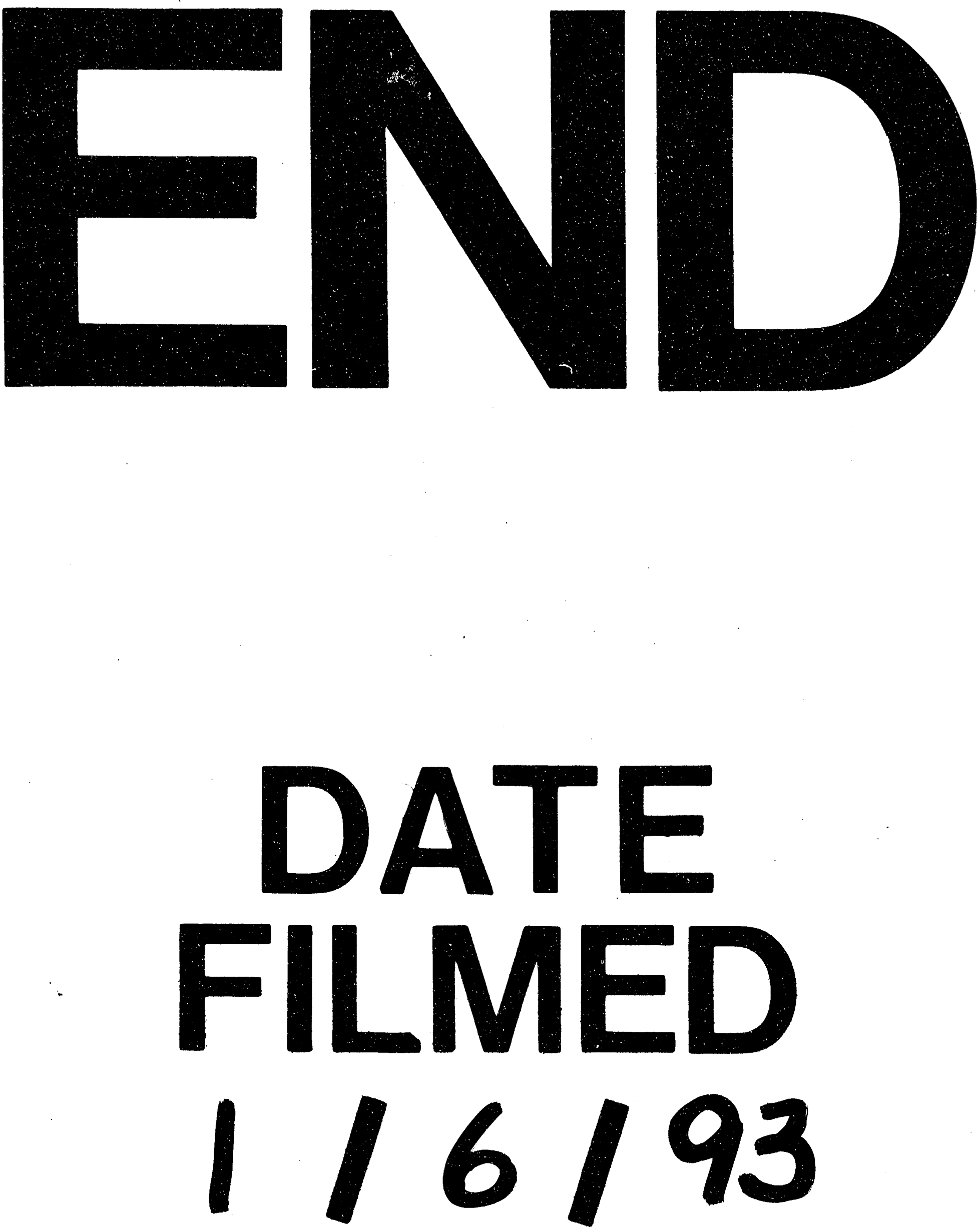
